\title{
ASSOCIAC̄̃̃ ENTRE O HÁBITO DE CONSUMO DE CAFÉ DA MANHÃ E ESTADO NUTRICIONAL DE ESTUDANTES UNIVERSITÁRIOS
}

\section{ASSOCIATION BETWEEN THE HABIT OF BREAKFAST CONSUMPTION AND THE NUTRITIONAL STATUS OF UNDERGRADUATE STUDENTS}

\author{
Anna Beatriz Silva Gomes ${ }^{1}$ \\ Rita de Cassia de Souza Fernandes ${ }^{2}$ \\ Alisson Diego Machado 3 \\ Edeli Simioni de Abreu 4 \\ Mônica Glória Neumann Spinelli5
}

\begin{abstract}
Resumo: O objetivo do estudo foi avaliar o hábito e a composição do café da manhã de jovens universitários, os fatores que influenciam na realização dessa refeição e sua associação com o estado nutricional. Como metodologia foi aplicado um questionário "online" disponibilizado em uma plataforma virtual de criação de formulários, contendo dados sociodemográficos (nome, idade, gênero, curso e o exercício de atividade remunerada); dados antropométricos (peso e estatura); questões específicas relacionadas ao consumo de café da manhã, como composição, frequência, horário e local de consumo e companhia; e fatores que influenciam na adoção dessa refeição. Foram analisados 143 universitários. Destes, 67,8\% possuem consumo regular de café da manhã, sendo mais consumidos alimentos in natura e minimamente processados. Observou-se que alunos da área biológica realizam o café da manhã com maior frequência do que os universitários das áreas de humanas e exatas. Dos participantes, 23,7\% possuíam excesso de peso. O estudo concluiu que apenas o ato de realizar ou não o café da manhã não está associado ao estado nutricional. O estado nutricional referente ao peso adequado se relacionou positivamente com o sexo feminino, não exercer atividade remunerada, satisfação corporal e realizar essa refeição sozinho, assim como a maior preocupação com excesso de peso foi relacionada às mulheres e o maior peso aos universitários que exerciam atividades remuneradas.
\end{abstract}

Palavras-chave: Hábitos alimentares; consumo de alimentos; desjejum; estudantes; estado nutricional.

Abstract: The study aimed to evaluate the habits and breakfast composition of young university students, the factors that influence the accomplishment of this meal and its association with the nutritional state. Methodology: Online questionnaire arranged in a virtual platform for the creation of forms, containing sociodemographic data (name, age, gender, course and the exercise of remunerated activity); anthropometric data (weight and height); specific questions related to breakfast consumption, such as composition, frequency, time and place of consumption and company; and factors that influence the adoption of this meal. Results and discussion: A total of 143 undergraduate students were analyzed. From this total, $67.8 \%$ have regular consumption of breakfast, with in natura and minimally processed foods being the most consumed. It was observed that students in the biological area have breakfast more frequently than university students in the human and exact areas. Among the participants, $23.7 \%$ were overweight. Conclusion: The study showed that only the act of making or not breakfast is not associated with the nutritional status. The nutritional status referring to the appropriate weight was positively correlated with the female gender, not to exercise remunerated activity, body satisfaction and to have this meal alone, as well as the greatest concern with overweight was correlated with women, and highest weight, with university students who exercised paid activities.

Keywords: Feeding behavior; food consumption; breakfast; students; nutritional status.

\footnotetext{
${ }^{1}$ Graduanda do curso de Nutrição no Centro de Ciências Biológicas e da Saúde da Universidade Presbiteriana Mackenzie, Brasil. E-mail: anna_8D@hotmail.com.

2 Graduanda do curso de Nutrição no Centro de Ciências Biológicas e da Saúde da Universidade Presbiteriana Mackenzie, Brasil. E-mail: ritadecsfernandes@gmail.com.

3 Mestre e doutorando em Ciências pelo Programa de Pós-Graduação em Nefrologia da Universidade de São Paulo, Brasil. E-mail: alissondiegomachado@hotmail.com.

4 Doutora pela Universidade de São Paulo. Docente do curso de graduação em Nutrição das Faculdades Metropolitanas Unidas (FMU), Brasil. E-mail: edelisabreu@gmail.com.

5 Doutora pela Universidade de São Paulo. Docente do curso de graduação em Nutrição no Centro de Ciências Biológicas e da Saúde da Universidade Presbiteriana Mackenzie, Brasil. E-mail: monicaspi404@gmail.com.
} 


\section{INTRODUÇÂO}

O atual Guia Alimentar para a População Brasileira inova no contexto da educação alimentar e nutricional por trazer conceitos e recomendações em linguagem didática para a população sobre um hábito alimentar saudável, qualidade nutricional, sustentabilidade e dimensões culturais e sociais das práticas alimentares. Em linguagem didática, descreve e ilustra a importância da realização das três principais refeições do dia (BRASIL, 2014). Nesse cenário, estudos têm referido que o café da manhã é considerado por muitos indivíduos a refeição mais importante e responsável pela garantia da ingestão de alimentos saudáveis e, quando ingerido em harmonia e variação, contribui para evitar excessos calóricos ao longo do dia e, também, pela manutenção do estado nutricional, por ser, conceitualmente, uma refeição com grande quantidade de vitaminas e minerais (UTTER et al., 2007; TRANCOSO; CAVALLI; PROENÇA, 2010; HÖFELMANN; MOMM, 2014).

O que se observa, entretanto, é que o tema alimentação tem encontrado novos e ingentes desafios, sendo a falta de tempo a principal dificuldade dos indivíduos em manterem um hábito alimentar adequado quantitativamente e qualitativamente (COUTINHO; GENTIL; TORAL, 2008). Nesse cenário, tem se destacado a alimentação dos estudantes universitários, que podem ter seus hábitos modificados nesse período, como a não realização do café da manhã, uma vez que se trata de um período de mudanças intensas e de busca de identidade pessoal e profissional (BORGES; LIMA FILHO, 2004).

Sabe-se que as escolhas alimentares são determinadas e sofrem influências por um conjunto de variáveis biológicas, demográficas, culturais e econômicas, condicionando de forma concomitante um processo dinâmico de transformações ao longo do tempo. Para esses estudantes, o ingresso no ensino superior envolve alterações de rotina, relacionamentos e ambiente. É comum a falta de recursos financeiros, distância considerável entre local de moradia, estudo e estágios e, também, oscilações emocionais, fatores estes que podem impactar em alterações nas escolhas dos alimentos e refletir negativamente no desempenho acadêmico. Para estudantes do período matutino, o desjejum pode ser prejudicado e é importante avaliar, conforme citam alguns estudos, riscos de desenvolvimento de carências ou excessos nutricionais (ENES; SILVA, 2009; ALMEIDA, 2005).

O fato de alguns morarem sozinhos ou não terem se adaptado ao ritmo da universidade, também exemplificam os motivos para a não realização do café da manhã por universitários (MAGALHÃES et al., 2002; ENES; SILVA, 2009; COTA; MIRANDA, 2006). De acordo com Almeida (2005), a preocupação com a imagem corporal, a prática de dietas restritivas e a ausência de fome também estão relacionados à diminuição no consumo desta refeição.

Como tem sido observado, um aumento da prevalência de obesidade em todo o mundo e, para o público universitário, além da alimentação, várias são as condições 
que podem refletir em aumento de peso, como a diminuição da prática de atividade física e o estresse (OMS; OPAN, 2003; HENRIQUES et al., 2012). O objetivo do presente trabalho é avaliar o hábito de consumo e composição do café da manhã de jovens universitários, os fatores que influenciam na realização dessa refeição e sua associação com o estado nutricional.

\section{METODOLOGIA}

Trata-se de um estudo exploratório descritivo de delineamento transversal, com abordagem quantitativa e qualitativa, sendo a população de estudo composta por amostra de conveniência com universitários maiores de dezoito anos, de ambos os sexos, sem restrição de raça e/ou curso, matriculados em instituições de ensino superior do Brasil.

A coleta de dados foi realizada por meio de um questionário online em uma plataforma virtual de criação de formulários, contendo dados sociodemográficos (nome, idade, gênero, curso e exercício de trabalho remunerado); dados antropométricos (peso e altura referidos); satisfação ou não com o peso atual; composição, frequência, horário e local de consumo do café da manhã e se realizavam a refeição ou não em companhia.

Foram incluídos e analisados como fatores que poderiam influenciar na adoção dessa refeição: a idade, sexo, exercer atividade remunerada, região do país em que mora, satisfação com o peso, morar sozinho ou em companhia e região do curso.

Na variável "área do curso", os alunos das áreas de humanas e exatas foram agrupados em uma única variável devido a uma maior concentração de alunos da área biológica.

A partir do peso e a altura referidos, foi calculado o Índice de Massa Corporal (IMC), por meio da divisão do peso $(\mathrm{kg})$ pela altura $(\mathrm{m})$ ao quadrado, sendo o estado nutricional dos participantes classificado no presente estudo como "peso adequado", quando o IMC esteve entre 18,5 e $24,9 \mathrm{~kg} / \mathrm{m}^{2}$ e "excesso de peso", quando maior e/ou igual a $25 \mathrm{~kg} / \mathrm{m}^{2}(\mathrm{WHO}, 1998)$.

Foi utilizada para avaliar o hábito de consumo de café da manhã, a classificação estipulada por Almeida (2005), na qual o café da manhã foi determinado como hábito regular quando consumido de 5 a 7 vezes por semana e irregular de 1 até 4 vezes nesse período.

Os dados quantitativos foram apresentados em mediana e intervalo interquartil e os dados qualitativos em frequências absoluta e relativa. Foram realizadas análises descritivas entre as variáveis de interesse e a realização de café da manhã e também para o estado nutricional.

As variáveis quantitativas foram avaliadas por meio do teste de Mann-Whitney e 
as categóricas pelo teste do qui-quadrado. Além disso, foram realizadas análises de regressão logística, bruta e ajustada, sendo a realização de café da manhã e o estado nutricional as variáveis dependentes. As análises foram realizadas no software SPSS versão 17.0 e considerou-se diferença significativa valores de $p<0,05$.

O presente estudo faz parte de um projeto maior intitulado "Avaliação das práticas alimentares de grupos populacionais e dos possíveis fatores de risco", com aprovação do Comitê de Ética em Pesquisa da Universidade Presbiteriana Mackenzie - CAAE: 49688715.4.0000.0084.

\section{RESULTADOS E DISCUSSÃO}

Foram entrevistados 143 universitários com idade mediana de 20 anos, sendo $79,0 \%$ do sexo feminino, assim como no trabalho de Reis, Silva Junior e Pinho (2014). Destes, $85,6 \%$ eram da Região Sudeste, 8,4\% da Região Centro-Oeste, 4,0\% do Nordeste, e 2,0\% do Sul. Representaram o curso de Nutrição 29,3\% dos estudantes $(\mathrm{N}=42)$.

Quanto ao consumo do café da manhã, obteve-se como resultado que 97 entrevistados $(67,8 \%)$ possuem um hábito regular, $6(4,2 \%)$ um hábito irregular e 40 $(28 \%)$ não o realizam.

Verificou-se na avaliação do estado nutricional que $23,7 \%$ possuíam excesso de peso, valor inferior aos encontrados por Carneiro et al. (2016) no Pará (41,3\%), mas semelhantes aos de Kretschmer et al. (2015) no Rio Grande do Sul (26,4\%). O IMC médio do grupo avaliado foi de $22,89 \pm 3,36 \mathrm{~kg} / \mathrm{m}^{2}$

A Tabela 1 contém uma análise descritiva, na qual não foram encontradas associações entre o hábito de realizar café da manhã com a idade, sexo, região do País, prática de atividade remunerada, morar sozinho ou com a família e quanto à satisfação do peso. O único dado que apresentou diferença significativa foi área do curso, sendo maior a frequência de realização do café da manhã por estudantes da área de exatas/humanas em comparação com a área biológica, sendo confirmado por meio da análise de regressão, exposto na Tabela 2. Quando comparadas a não realização, maior rejeição é observada na área de exatas/humanas (70\%).

Tabela 1 - Associação entre o hábito de realizar o café da manhã e variáveis sociodemográficas e de estilo de vida de estudantes universitários no ano de 2017.

(continuação).

\begin{tabular}{lccc}
\hline \multirow{2}{*}{ Variável } & \multicolumn{2}{c}{ Consumo de café da manhã } & \multirow{2}{*}{$\mathbf{p}$} \\
\cline { 2 - 3 } & Sim & Não & 0,23 \\
Sexo & & & \\
$\quad$ Feminino & $84(81,6 \%)$ & $29(72,5 \%)$ & \\
Masculino & $19(18,4 \%)$ & $11(27,5 \%)$ & \\
Área do curso & & & $\mathbf{0 , 0 5}$ \\
$\quad$ Biológicas & $50(48,5 \%)$ & $12(30,0 \%)$ & \\
\hline
\end{tabular}


Tabela 1 - Associação entre o hábito de realizar o café da manhã e variáveis sociodemográficas e de estilo de vida de estudantes universitários. (conclusão)

\begin{tabular}{lccc}
\hline Exatas/Humanas & $53(51,5 \%)$ & $28(70,0 \%)$ & 0,95 \\
Região & & & \\
$\quad$ Sudeste & $88(85,4 \%)$ & $34(85,0 \%)$ & \\
$\quad$ Demais regiões & $15(14,6 \%)$ & $6(15,0 \%)$ & 0,25 \\
$\begin{array}{l}\text { Exerce atividade remunerada } \\
\text { Não }\end{array}$ & $56(54,4 \%)$ & $26(65,0 \%)$ & \\
$\quad$ Sim & $47(45,6 \%)$ & $14(35,0 \%)$ & \\
Com quem mora & & $35(87,5 \%)$ & 0,75 \\
$\quad$ Família & $88(85,4 \%)$ & $5(12,5 \%)$ & \\
$\quad$ Sozinho/Amigos & $15(14,6 \%)$ & & 0,88 \\
Satisfação com o peso & & $25(62,5 \%)$ & \\
$\quad$ Não & $63(61,2 \%)$ & $15(37,5 \%)$ & \\
Sim & $40(38,8 \%)$ &
\end{tabular}

Fonte: Os autores.

Tabela 2 - Regressão logística bruta e ajustada: Associação entre o hábito de realizar o café da manhã e variáveis sociodemográficas e de estilo de vida de estudantes universitários.

\begin{tabular}{|c|c|c|c|c|}
\hline Variável & $\begin{array}{c}\text { OR (IC95\%) } \\
\text { Bruto }\end{array}$ & $\mathbf{P}$ & $\begin{array}{c}\text { OR (IC95\%) } \\
\text { Ajustado }\end{array}$ & $\mathbf{p}$ \\
\hline Idade & $1,16(0,97-1,37)$ & 00,10 & & \\
\hline \multicolumn{5}{|l|}{ Sexo } \\
\hline Feminino & 1 & & & \\
\hline Masculino & $0,60(0,25-1,40)$ & 0,24 & & \\
\hline \multicolumn{5}{|l|}{ Área do curso } \\
\hline Biológicas & 1 & & 1 & \\
\hline Exatas/Humanas & $0,45(0,21-0,99)$ & 00,05 & $0,38(0,17-0,85)$ & $0,02^{a}$ \\
\hline \multicolumn{5}{|l|}{ Região } \\
\hline Sudeste & 1 & & & \\
\hline Demais regiões & $0,97(0,35-2,70)$ & 0,95 & & \\
\hline \multicolumn{5}{|c|}{ Exerce atividade remunerada } \\
\hline Não & 1 & & & \\
\hline Sim & $1,56(0,73-3,32)$ & 0,25 & & \\
\hline \multicolumn{5}{|l|}{ Com quem mora } \\
\hline Família & 1 & & & \\
\hline Sozinho/Amigos & $1,19(0,40-3,53)$ & 0,75 & & \\
\hline \multicolumn{5}{|c|}{ Satisfação com o peso } \\
\hline Não & 1 & & & \\
\hline Sim & $1,06(0,50-2,25)$ & 0,88 & & \\
\hline
\end{tabular}

Fonte: Os autores.

A Tabela 3 apresenta a relação entre o estado nutricional dos estudantes (peso adequado e excesso de peso) com as variáveis questionadas, apresentando associação com o sexo dos indivíduos, prática de atividade remunerada, satisfação corporal e em relação a tomar café da manhã acompanhado ou sozinho. Nas análises de regressão, mesmo após ajustes, essas variáveis também se mostraram associadas, conforme Tabela 4. Com relação às demais variáveis não foram encontradas associações estatisticamente significativas. 
Tabela 3 - Variáveis relacionadas ao estado nutricional de estudantes universitários.

\begin{tabular}{|c|c|c|c|}
\hline \multirow{2}{*}{ Variável } & \multicolumn{2}{|c|}{ Estado nutricional } & \multirow{2}{*}{$\mathbf{p}$} \\
\hline & Peso adequado & Excesso de peso & \\
\hline Idade & $20(19-21)$ & $20(19-22)$ & 0,64 \\
\hline Sexo & & & 0,003 \\
\hline Feminino & $93(84,5 \%)$ & $20(60,6 \%)$ & \\
\hline Masculino & $17(15,5 \%)$ & $13(39,4 \%)$ & \\
\hline Área & & & 0,60 \\
\hline Biológicas & $49(44,5 \%)$ & $13(39,4 \%)$ & \\
\hline Exatas/Humanas & $61(55,5 \%)$ & $20(60,6 \%)$ & \\
\hline Região & & & 0,64 \\
\hline Sudeste & $93(84,5 \%)$ & $29(87,9 \%)$ & \\
\hline Demais regiões & $17(15,5 \%)$ & $4(12,1 \%)$ & \\
\hline Exerce atividade remunerada & & & 0,005 \\
\hline Não & $70(63,6 \%)$ & $12(36,4 \%)$ & \\
\hline Sim & $40(36,4 \%)$ & $21(63,6 \%)$ & \\
\hline Com quem mora & & & 0,43 \\
\hline Família & $96(87,3 \%)$ & $27(81,8 \%)$ & \\
\hline Sozinho/Amigos & $14(12,7 \%)$ & $6(18,2 \%)$ & \\
\hline Satisfação com o peso & & & 0,02 \\
\hline Não & $62(56,4 \%)$ & $26(78,8 \%)$ & \\
\hline Sim & $48(43,6 \%)$ & $7(21,2 \%)$ & \\
\hline Realiza café da manhã & & & 0,73 \\
\hline Não & $30(27,3 \%)$ & $10(30,3 \%)$ & \\
\hline Sim & $80(72,7 \%)$ & $23(69,7 \%)$ & \\
\hline Frequência & & & 0,35 \\
\hline 1-4 vezes/semana & $5(6,2 \%)$ & $1(4,3 \%)$ & \\
\hline 5-6 vezes/semana & $17(21,3 \%)$ & $2(8,7 \%)$ & \\
\hline Diariamente & $58(72,5 \%)$ & $20(87,0 \%)$ & \\
\hline Com quem realiza o café da manhã & & & 0,05 \\
\hline Acompanhado & $21(26,2 \%)$ & $11(47,8 \%)$ & \\
\hline Sozinho & $59(73,8 \%)$ & $12(52,2 \%)$ & \\
\hline Local & & & 0,51 \\
\hline Casa & $57(71,2 \%)$ & $18(78,3 \%)$ & \\
\hline Outros & $23(28,8 \%)$ & $5(21,7 \%)$ & \\
\hline
\end{tabular}

Fonte: Os autores.

Tabela 4 - Regressão logística bruta e ajustada: Variáveis relacionadas ao estado nutricional de estudantes universitários.

\begin{tabular}{|c|c|c|c|c|}
\hline Variável & $\begin{array}{c}\text { OR (IC95\%) } \\
\text { Bruto }\end{array}$ & $\mathbf{P}$ & $\begin{array}{l}\text { OR (IC95\%) } \\
\text { Ajustado }\end{array}$ & $\mathbf{p}$ \\
\hline Idade & $1,05(0,97-1,14)$ & 0,22 & & \\
\hline Sexo & & & & \\
\hline Feminino & 1 & & & \\
\hline Masculino & $3,56(1,49-8,48)$ & 0,004 & & \\
\hline Área & & & & \\
\hline Biológicas & 1 & & & \\
\hline Exatas/Humanas & $1,24(0,56-2,73)$ & 0,60 & & \\
\hline Região & & & & \\
\hline Sudeste & 1 & & & \\
\hline Demais regiões & $0,76(0,24-2,42)$ & 0,64 & & \\
\hline Exerce atividade remunerada & & & & \\
\hline Não & 1 & & 1 & \\
\hline Sim & $3,06(1,36-6,88)$ & 0,01 & $2,43(1,04-5,67)$ & $0,04^{a}$ \\
\hline Com quem mora & & & & \\
\hline Família & 1 & & & \\
\hline $\begin{array}{l}\text { Sozinho/Amigos } \\
\text { Satisfação com o peso }\end{array}$ & $1,52(0,54-4,34)$ & 0,43 & & \\
\hline
\end{tabular}


Tabela 4 - Regressão logística bruta e ajustada: Variáveis relacionadas ao estado nutricional de estudantes universitários.

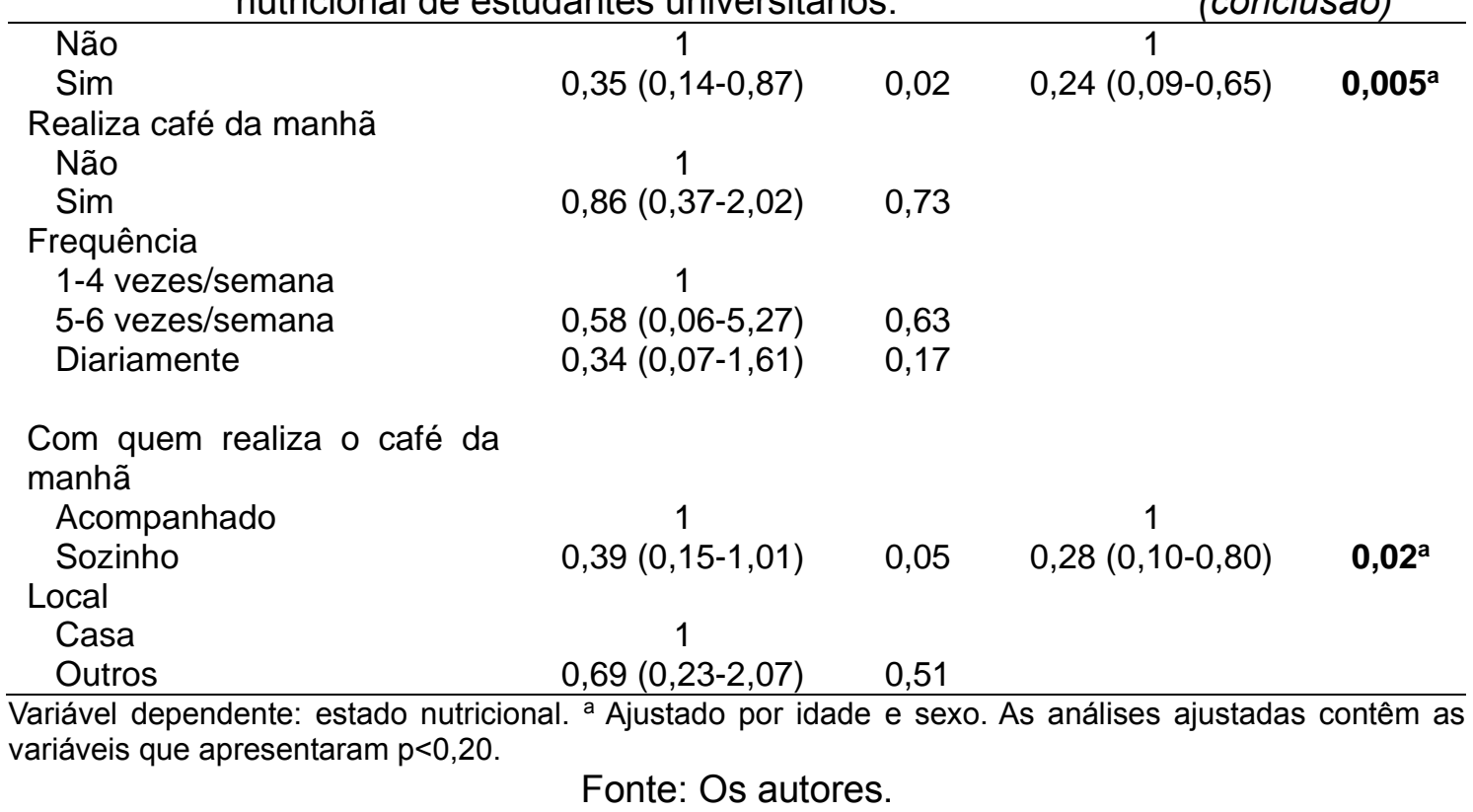

Demonstrou-se, com esses resultados, a possibilidade de haver uma maior preocupação com excesso de peso por parte do público feminino do que o público masculino, o que também foi relatado por Araújo e Araújo (2003) em outro estudo, que detectou que as mulheres desejavam reduzir mais o seu peso corporal do que os homens e que analisou também a autopercepção e insatisfação com peso corporal, comparando as diferenças entre peso relatado e peso desejado, em ambos os sexos. Foi possível observar que os universitários que exercem atividade remunerada apresentaram maior excesso de peso, resultado diferente do exposto por Melo, Batista Filho e Rissin (2015), em que 68,8 \% dos indivíduos que não trabalhavam apresentavam sobrepeso $(28,8 \%)$ ou obesidade $(40,0 \%)$.

Ainda foi encontrado que universitários do sexo masculino têm 3,5 mais chances de ter sobrepeso do que o sexo feminino, podendo o sedentarismo ser uma das causas para esse aumento, visto que o estudo de Andrade et al. (2015) identificou que homens com escolaridade superior a 12 anos e residentes no setor de maior tercil de renda exercem, em sua maioria, atividades sedentárias.

Houve relato de uma variedade muito grande de alimentos no desjejum. Dos mais consumidos, no café da manhã dos universitários, houve destaque para o leite (30,7\%), seguido das frutas $(27,2 \%)$, manteiga e margarina $(19,5 \%)$ e pães $(15,3 \%)$. Mattos e Martins (2000), com amostra de 559 indivíduos com mais de 20 anos do município de Cotia (SP), identificou os mesmos alimentos em sua pesquisa como o pão francês $(70,8 \%)$, o leite $(51,3 \%)$ e margarina $(50,8 \%)$, mas com predomínio do café $(87,5 \%)$, sendo o consumo de frutas referido por apenas $11,3 \%$ dessa população. 


\section{CONSIDERAÇÕES FINAIS}

Com o presente estudo foi possível observar que na amostra avaliada, composta principalmente por estudantes mulheres, da Região Sudeste e da área biológica, o hábito regular do consumo de café da manhã foi relatado por $67,8 \%$ dos participantes do estudo. É válido notar, entretanto, um percentual importante de universitários que não consomem o café da manhã, o que pode impactar em diminuição do desempenho acadêmico.

Foi possível notar na pesquisa que o único fator de influência à realização dessa refeição está relacionado a área do curso ao qual o universitário está matriculado, pois houve maior adesão dos estudantes da área de biológicas, identificando-se também que há $55 \%$ a mais de chance de estudantes de humanas e exatas não realizarem o café da manhã por serem dessas áreas.

O estudo identificou que $23,7 \%$ dos participantes possuíam excesso de peso, sendo que apenas o ato de realizar ou não o café da manhã não se apresentou associado ao estado nutricional quanto a manter um peso adequado; tendo em vista esse resultado, pode-se inferir como hipótese que não apenas o consumo, mas o conjunto das escolhas alimentares dos indivíduos para essa refeição podem influenciar no estado nutricional do indivíduo.

O estado nutricional referente ao peso adequado se relacionou positivamente com o sexo feminino por meio dos dados: não exercer atividade remunerada, satisfação corporal e fazer essa refeição sozinho, assim como a maior preocupação com excesso de peso; todavia, o maior peso se relacionou aos universitários que exerciam atividades remuneradas.

\section{REFERÊNCIAS}

ALMEIDA, E. C. Hábito de consumo do café da manhã por adolescentes estudantes de escolas públicas e privadas, localizadas na região central da cidade de Cotia. 2005. Tese (Mestrado) - Universidade Federal de São Paulo, São Paulo, 2005.

ANDRADE, R. G. et al. Overweight in men and women among urban area residents: individual factors and socioeconomic context. Cadernos de Saúde Pública, v. 31, suppl. 1, p. 148-158, 2015.

ARAÚJO, D. S. M. S.; ARAÚJO, C. G. S. Autopercepção e insatisfação com peso corporal independem da frequência de atividade física. Arquivos Brasileiros de Cardiologia, v. 80, n. 3, p. 235-242, 2003.

BORGES, C. M.; LIMA FILHO, D. O. Hábitos alimentares dos estudantes universitários: um estudo qualitativo. In: SEMEAD, 7., 2004. Anais..., Mato Grosso do Sul, 2004. 
BRASIL. Ministério da Saúde. Secretaria de Atenção à Saúde. Departamento de Atenção Básica. Guia alimentar para a população brasileira. 2. ed. Brasília: Ministério da Saúde, 2014. 156 p.

CARNEIRO, M. Z. L. et al. Estado nutricional de estudantes universitários associados aos hábitos alimentares. Revista da Sociedade Brasileira de Clínica Médica, v. 14, n. 2, p. 84-88, 2016.

COTA, R. P.; MIRANDA, L. S. Associação entre constipação intestinal e estilo de vida em estudantes universitários. Revista Brasileira de Nutrição Clínica, v. 21, n. 4, p. 296-301, 2006.

COUTINHO, J. G.; GENTIL, P. C.; TORAL, N. A desnutrição e obesidade no Brasil: o enfrentamento com base na agenda única da nutrição. Cadernos de Saúde Pública, v. 24 , n. 2 , p. $332-40,2008$.

ENES, C. C.; SILVA, M. V. Disponibilidade de energia e nutrientes nos domicílios: o contraste entre as regiões Norte e Sul do Brasil. Ciência e Saúde Coletiva, v. 14, n. 4, p. 1267-1276, 2009.

HENRIQUES, P. et al. Regulamentação da propaganda de alimentos infantis como estratégia para a promoção da saúde. Ciência e Saúde Coletiva, v. 17, n. 2, p. 48190, 2012.

HÖFELMANN, D. A.; MOMM, N. Café da manhã: omissão e fatores associados em escolares de Itajaí, Santa Catarina, Brasil. Nutrire: Revista da Sociedade Brasileira de Alimentação e Nutrição, v. 39, n. 1, p. 40-55, 2014.

KRETSCHMER, A. C. et al. Estado nutricional e hábitos alimentares de acadêmicos de uma universidade do norte do Rio Grande do Sul. Revista Saúde (Santa Maria), v. 41, n. 2, p. 121-128, 2015.

MAGALHÃES, A. C. P. et al. Prevalência e fatores de risco para constipação intestinal em estudantes universitários. Revista Nutrição em Pauta. n. 56, p. 13-17, 2002.

MATOS, L. L.; MARTINS, I.S. Consumo de fibras alimentares em população adulta. Revista de Saúde Pública, v. 34, n. 1, p. 50-55, 2000.

MELO, S. P. S. C.; BATISTA FILHO, M.; RISSIN, A. Excesso de peso de adultos residentes em um aglomerado urbano subnormal. Revista Brasileira em Promoção da Saúde, v. 28, n. 2, p. 257-265, 2015.

ORGANIZAÇÃO MUNDIAL DE SAÚDE - OMS; ORGANIZAÇÃO PAN AMERICANA DE SAÚDE - OPAN. Doenças Crônicas - Degenerativas e Obesidade: estratégia mundial sobre alimentação saudável, atividade física e saúde. Brasília, 2003.

REIS, J. A.; SILVA JÚNIOR, C. R. R.; PINHO, L. Fatores associados ao risco de transtornos alimentares entre acadêmicos da área de saúde. Revista Gaúcha de Enfermagem, v. 35, n. 2, p. 73-78, 2014.

TRANCOSO, S. C.; CAVALLI, S. B.; PROENCA, R. P. da C. Café da manhã: caracterização, consumo e importância para a saúde. Revista de Nutrição, v. 23, n. 5, p. 859-869, 2010. 
UTTER, J. et al. At-home breakfast consumption among New Zealand children: Associations with body mass index and related nutrition behaviors Journal of the American Dietetic Association, v.107, n. 4, p. 570-576, 2007.

WORLD HEALTH ORGANIZATION - WHO. Obesity - preventing and managing the global epidemic. Report of a WHO consultation on obesity. Geneva, 1998. 